Supporting Information for

\title{
Enhanced In-source Fragmentation Annotation Enables Novel Data Independent Acquisition and Autonomous METLIN Molecular Identification
}

\author{
Jingchuan Xue ${ }^{1, \#}$, Xavier Domingo-Almenara ${ }^{2, \#}$, Carlos Guijas ${ }^{1}$, Amelia Palermo ${ }^{1}$, Markus M \\ Rinschen ${ }^{1}$, John Isbell ${ }^{3}$, H. Paul Benton ${ }^{1}$, and Gary Siuzdak ${ }^{1, *}$
}

${ }^{1}$ Scripps Center for Metabolomics and Department of Molecular and Computational Biology, The Scripps Research Institute, 10550 North Torrey Pines Road, La Jolla, CA 92037, United States

${ }^{2}$ Centre for Omic Sciences, EURECAT - Technology Centre of Catalonia \& Rovira i Virgili University joint unit, Reus, Catalonia, Spain

${ }^{3}$ Discovery Chemistry, Genomics Institute of the Novartis Research Foundation, Metabolism and Pharmacokinetics, San Diego, California 92121, United States

\#authors contributed equally

*Corresponding author

Gary Siuzdak, PhD

Scripps Center for Metabolomics

The Scripps Research Institute, La Jolla, CA 92037

Tel.: 8587849113

Email: siuzdak@scripps.edu

Table of Contents

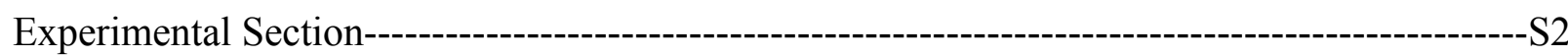

In-Source Fragmentation Condition Optimization Results Section-----------------------------S5

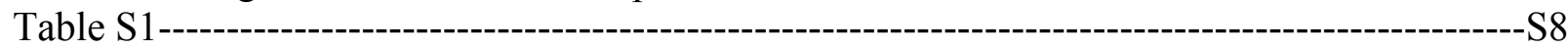

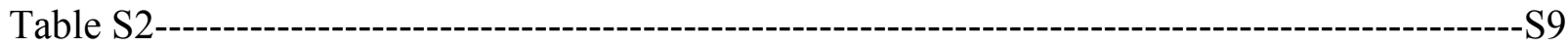

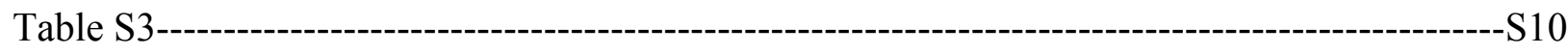

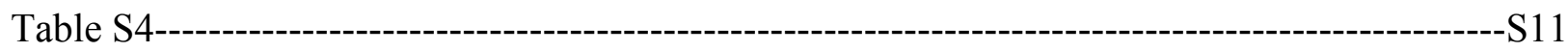

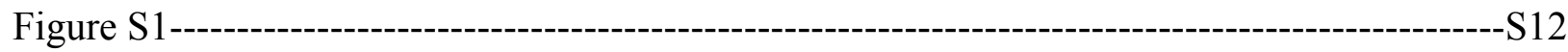

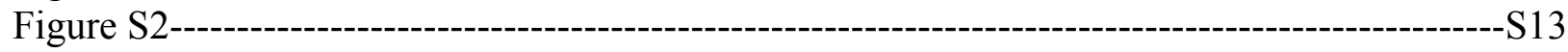

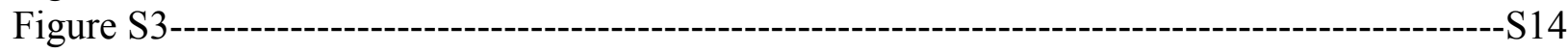




\section{Experimental Section}

Materials. For the optimization of in-source fragmentation conditions, a mixture of 50 endogenous metabolites was prepared in water at $30 \mu \mathrm{M}$. These metabolites were selected to represent a broad range of physicochemical properties and metabolic pathways, such as amino acids, lipids, citric acid cycle, nucleotides, and coenzyme. For the investigation of sensitivity, a mixture of four metabolites in each ionization mode (tyrosine, inosine, uridine monophosphate (UMP), and oxidized glutathione in negative mode; phenylalanine, glutamine, histidine, and oxidized glutathione at positive mode) was prepared at 9 concentrations ranging 6 orders of magnitude: $1 \mathrm{nM}, 10 \mathrm{nM}, 100 \mathrm{nM}, 1 \mu \mathrm{M}, 10 \mu \mathrm{M}, 50 \mu \mathrm{M}, 100 \mu \mathrm{M}, 500 \mu \mathrm{M}$, and $1 \mathrm{mM}$. The standards were purchased from Sigma-Aldrich (St. Louis, MO).

Metabolite extraction. Metabolites in the macrophage samples were extracted using the method described elsewhere with slight modifications. ${ }^{1}$ In brief, cell samples were sonicated in ice for 15 mins after a shock-freezing in liquid nitrogen and subsequent thawing at room temperature. The operation was repeated for three times. Then the sample was incubated at $-20{ }^{\circ} \mathrm{C}$ overnight for protein precipitation, followed by centrifugation at $13,000 \mathrm{rpm}$ and $4{ }^{\circ} \mathrm{C}$ for $15 \mathrm{mins}$. The supernatant was dried in a vacuum concentrator and reconstituted with $50 \mu \mathrm{L}$ acetonitrile:water (v:v; 1:1). After sonication (10 mins, in ice) and centrifugation $\left(13,000 \mathrm{rpm}, 4^{\circ} \mathrm{C}, 15 \mathrm{mins}\right)$, the supernatant was transferred to a LC-MS glass vial for instrumental injection.

High-Resolution Mass Spectrometry Analysis. The metabolite mixture was analyzed with a liquid chromatography electrospray ionization quadrupole time-of-flight mass spectrometry (LCESI-QTOF-MS) (BRUKER impact II) in both positive and negative ESI modes. ZORBAX 300 SB-C18 column $(0.5 \times 150 \mathrm{~mm}, 5 \mu \mathrm{m}$, Agilent $)$ and Luna ${ }^{\circledR} \mathrm{NH} 2100 \AA$ A column $(1 \times 150 \mathrm{~mm}, 3$ $\mu \mathrm{m}$, Phenomenex) were used in the separation of metabolites in reverse phase (positive mode) and HILIC analysis (negative mode), respectively. For the reverse phase analysis, metabolites were separated by gradient elution at a flow rate of $20 \mu \mathrm{L} / \mathrm{min}$ starting at $5 \%(\mathrm{v} / \mathrm{v}) \mathrm{B}$, held for 5 min, increased to $95 \% \mathrm{~B}$ within $45 \mathrm{~min}$, held for $5 \mathrm{~min}$, and reverted to $5 \% \mathrm{~B}$ at $56^{\text {th }}$ min, held for $5 \mathrm{~min}$, with a total run time of $61 \mathrm{~min}$. The mobile phases comprised water containing $0.1 \%$ formic acid (A) and acetonitrile containing $0.1 \%$ formic acid (B). For the HILIC analysis, metabolites were separated by gradient elution at a flow rate of $50 \mu \mathrm{L} / \mathrm{min}$ starting at $0 \%$ (v/v) A, 
held for $1 \mathrm{~min}$, increased to $100 \% \mathrm{~A}$ within $47 \mathrm{~min}$, held for $5 \mathrm{~min}$, and reverted to $0 \% \mathrm{~A}$ at $56^{\text {th }}$ min, held for $5 \mathrm{~min}$, with a total run time of $61 \mathrm{~min}$. The mobile phases comprised water/acetonitrile (v/v; 95:5) containing $20 \mathrm{mM}$ ammonium acetate and $40 \mathrm{mM}$ ammonium hydroxide (A) and water/acetonitrile (v/v; 5:95) (B).

The original ESI source parameters were set as follows: dry temperature $180^{\circ} \mathrm{C}$, dry gas 7 $\mathrm{L} / \mathrm{min}$, nebulizer $20.3 \mathrm{psi}$, and capillary voltage $4500 \mathrm{~V}$ and $-4000 \mathrm{~V}$ for positive and negative modes, respectively. The transfer parameters were: funnel 1 RF 150 Vpp, funnel 2 RF 200 Vpp, isCID energy $0 \mathrm{eV}$, and hexapole RF $50 \mathrm{Vpp}$. The ion energy in the quadrupole was set to $4 \mathrm{eV}$ and the collision energy applied in the collision cell was set at $7 \mathrm{eV}$. The instrument acquisition range was set at $50-1200 \mathrm{~m} / \mathrm{z}$ and the MS acquisition rate was 2 spectra/s.

As discussed in the results section, transfer isCID energy was optimized to increase the insource fragmentation while maintaining the intensity of precursor ions. After optimization, $30 \mathrm{eV}$ and $40 \mathrm{eV}$ transfer isCID energy were selected as the enhanced in-source fragmentation condition in positive and negative mode, respectively, with all other parameters fixed.

For the data dependent acquisition (DDA) of MS/MS spectra, both the MS and MS/MS acquisition rates were set at 2 spectra/s to acquire over the $m / z$ range $50-1200$. The collision energy was fixed at $20 \mathrm{eV}$ for all the molecules, since a large number of small molecules generate specific fragments at $20 \mathrm{eV}$.

For the data independent acquisition (DIA) of MS/MS spectra, Broadband Collision Induced Dissociation (bbCID) mode was selected with low and high collision energy as 7 and 20 $\mathrm{eV}$, respectively. isCID energy was set at $0 \mathrm{eV}$ for both channel 1 (low collision energy) and 2 (high collision energy). The acquisition factors were set at 1 for both high and low collision energy. The low collision energy aims to provide full scan TOF MS spectra and the high collision energy is to generate the DIA MS/MS spectra for all the peaks without precursor isolation. The $\mathrm{m} / \mathrm{z}$ range was set as $50-1200$.

\section{Single Quadrupole Mass Spectrometry Analysis. An Agilent InfinityLab Liquid} Chromatography/Mass Selective Detector (LC-MSD) system was used in the single quadrupole MS based untargeted experiments. Poroshell 120 EC-C8 column (4.6 x 50 mm, $2.7 \mu \mathrm{m}$, Agilent) was used in the analysis of metabolite mixture in the positive mode; Acquity BEH Amide column ( 1 x $100 \mathrm{~mm}, 1.7 \mu \mathrm{m}, 130 \AA$, Waters) was used in the negative mode. The gradient in 
positive mode was as follows: starting at 5\% (v/v) B, held for $2 \mathrm{~min}$, increased to $95 \% \mathrm{~B}$ within $12 \mathrm{~min}$, held for $2.5 \mathrm{~min}$, and reverted to $5 \% \mathrm{~B}$ at $17^{\text {th }} \mathrm{min}$, held for $3 \mathrm{~min}$, with a total run time of $20 \mathrm{~min}$; mobile phase $\mathrm{A}$, water containing $0.1 \%$ formic acid (A); mobile phase $\mathrm{B}$, acetonitrile containing $0.1 \%$ formic acid; mobile phase flow rate, $500 \mu \mathrm{L} / \mathrm{min}$. The gradient in negative mode was as follows: starting at 1\% (v/v) A, held for $1 \mathrm{~min}$, increased to 35\% A within 13 min, then increased to $60 \% \mathrm{~A}$ at $17^{\text {th }} \mathrm{min}$, held for $1 \mathrm{~min}$, and reverted to $1 \% \mathrm{~A}$ at $19^{\text {th }} \mathrm{min}$, held for 6 min, with a total run time of 25 min; mobile phase A, water:acetonitrile (v:v; 95:5) containing 5 $\mathrm{mM}$ ammonium acetate and $5 \mathrm{mM}$ ammonium hydroxide; mobile phase $\mathrm{B}$, water:acetonitrile (v:v; 5:95); mobile phase flow rate, $100 \mu \mathrm{L} / \mathrm{min}$.

The ESI source parameters were set as follows: in positive mode, drying gas flow 12 $\mathrm{L} / \mathrm{min}$, drying gas temperature $350^{\circ} \mathrm{C}$, nebulizer pressure $35 \mathrm{psig}$, and capillary voltage $4000 \mathrm{~V}$; in negative mode, drying gas flow $8 \mathrm{~L} / \mathrm{min}$, drying gas temperature $325^{\circ} \mathrm{C}$, nebulizer pressure 30 psig, and capillary voltage $3500 \mathrm{~V}$. Mass spectra was acquired over the $\mathrm{m} / \mathrm{z}$ range of 50-1200 at two fragmentor voltages, 150 and $300 \mathrm{~V}$, respectively.

Data Analysis. LC-MS and MS/MS datasets generated under different conditions were manually inspected with the Bruker Compass Data Analysis software (Version 4.4) to group the features (precursors and fragments) stemming from the same metabolite in high resolution MS based untargeted experiments. ChemStation software was used in the data analysis in single quadrupole MS based untargeted experiments. The targeted ion extraction window was $\pm 0.01 \mathrm{~m} / \mathrm{z}$ in high resolution MS data analysis and $(-0.3,+0.7)$ da in single quadrupole MS data analysis.

eISA approach considers each molecule in the spectral library (e.g., METLIN) as an analysis target. By using this metabolite-centric approach, direct link between precursor ion and the fragments was established in the $\mathrm{MS}^{1}$ data produced by eISA technique. The molecular identification procedure using eISA approach was shown as follows: (1) search the precursor ion $\mathrm{m} / \mathrm{z}$ in the $\mathrm{MS}^{1}$ chromatogram generated by eISA within a predefined window (e.g., $\pm 0.01 \mathrm{~m} / \mathrm{z}$ in high resolution MS data); (2) carefully select the peak of the precursor ion based on the retention time and acquire the compound spectra at the highest point of the peak; (3) record the in-source fragments of the precursor ion that matched to the reference spectral library based on the predefined mass accuracy criteria (e.g., $50 \mathrm{ppm}$ in high resolution MS data); (4) evaluate the fragmentation pattern similarity between eISA technique and other techniques using two 
matching scores, ratio score and match factor; ${ }^{2}(5)$ assign putative molecular identification based on the fragmentation pattern similarity along with other orthogonal information (e.g., retention time). In this study, the MS/MS spectra generated at $20 \mathrm{eV}$ in the METLIN library were used as reference spectra in molecular identification. Two indicators used in the molecular identification, ratio score and match factor, have been explained in details in our earlier study. ${ }^{2}$ Briefly, ratio score was calculated as a fraction, with the denominator as the total number of fragments in the $20 \mathrm{eV}$ MS/MS spectra in the METLIN library, unless stated otherwise. The numerator was the number of fragment ions in $\mathrm{MS}^{1}$ (generated with eISA or DIA) or MS/MS (generated with DDA mode) that matched to the denominator. Match factor was calculated using the cosine dot product, a specific mathematical approach widely used in the determination of mass spectral similarity. ${ }^{3}$ The match factor was calculated only for those metabolites with 3 or more fragments in their spectra. Match factor ranged from 0 to $100(\%)$, with 100 being the highest similarity match. Precursors were excluded from the computation of both ratio score and match factor. Further, only those fragments with over 5\% relative intensity in the $20 \mathrm{eV} \mathrm{MS} / \mathrm{MS}$ spectra in the METLIN database were used, unless all the fragments have relative intensities below 5\% at 20 $\mathrm{eV}$. The fragments outside the mass acquisition range (below $50 \mathrm{~m} / \mathrm{z}$ ) were not considered. Statistical analysis was conducted with GraphPad Prism 8.

\section{In-Source Fragmentation Condition Optimization Results Section}

This study aimed to find in-source fragmentation conditions in which more fragments can be generated without compromising the intensity of precursor ions (Fig. 1). Specifically, the aim was to generate mass spectral data including both the high abundance precursor ions and their corresponding in-source fragments for confident compound identification within a single run in full scan mode. The $20 \mathrm{eV}$ MS/MS spectra in the METLIN library, produced in the collision cell with analytical standards, was used as a reference for the optimization process. We started with optimizing the electrospray ionization (ESI) source parameters such as capillary voltage and dry gas temperature to increase in-source fragmentation. Capillary voltage is known to control the desolvation/activation energy, which is directly related with the in-source fragmentation. ${ }^{4,5}$ One purpose of dry gas within the source is to aid desolvation in electrospray source. Higher capillary voltage and dry gas temperature can facilitate the generation of in-source fragments. ${ }^{4,5}$ In the BRUKER impact II, the suggested range of capillary voltage is 2000 to $5000 \mathrm{~V}$ in positive mode 
and 1500 to $4000 \mathrm{~V}$ in negative mode, respectively; the suggested range of dry gas temperature is 180 to $240{ }^{\circ} \mathrm{C}$, and the capillary voltage was set at $4500 \mathrm{~V}$ in positive mode and $4000 \mathrm{~V}$ in negative mode, respectively; the dry gas temperature was set up as $180^{\circ} \mathrm{C}$. Thus, we first increased the dry gas temperature to $240{ }^{\circ} \mathrm{C}$ to observe the impact on in-source fragmentation. As exemplified with four metabolites in Table S1, 1-palmitoyl-sn-glycero-3-phosphocholine and cytidine monophosphate in the positive mode and uridine and glucose-6-phosphate in the negative mode, increasing dry gas temperature does not significantly impact in-source fragmentation.

Next, we focused on the optimization of energy between ion funnel 1 and ion funnel 2, called the transfer isCID energy. The $\mathrm{MS}^{1}$ spectra for the above mentioned four metabolites at two transfer isCID energies ( 20 and $40 \mathrm{eV}$ ) are shown in Table $\mathrm{S} 1$, where increasing transfer isCID energy from 0 (original setting) to $40 \mathrm{eV}$ significantly improved the in-source fragmentation of selected molecules in both ionization modes.

To test the generality of this observation and find the appropriate transfer isCID energy for in-source fragmentation, we analyzed a mixture of 50 endogenous metabolites $(30 \mu \mathrm{M})$ by varying transfer is CID (from 0 to $100 \mathrm{eV}$ in $10 \mathrm{eV}$ increments). These metabolites were all observed in negative mode and their MS/MS spectra at $-20 \mathrm{eV}$ were all available in the METLIN library, however, in positive mode, only 33 metabolites were observed. Three factors were mainly considered in selecting the appropriate ISF condition: the number and relative intensity of fragments that matched to $20 \mathrm{eV}$ MS/MS spectra in the METLIN database and the intensity of precursor ion.

To assess the in-source fragmentation performance, we first calculated the percentage of fragments of each metabolite generated at each condition versus the total number of fragments recorded in the $20 \mathrm{eV} \mathrm{MS/MS} \mathrm{spectra} \mathrm{in} \mathrm{the} \mathrm{METLIN} \mathrm{database.} \mathrm{Only} \mathrm{those} \mathrm{fragments} \mathrm{with} \mathrm{a}$ relative intensity above 5\% were considered. As shown in Fig. S1a and S1b, the median fragment percentage observed in both positive and negative modes increased with the increase of isCID energy. A similar trend was also observed for match factor (Fig. S1c and S1d), which was calculated for those metabolites with at least 3 fragments in the $20 \mathrm{eV} \mathrm{MS/MS} \mathrm{spectra} \mathrm{in} \mathrm{the}$ METLIN database to assess the relative intensity similarity. The relationship between the median value of $1^{\text {st }}$ top fragment intensity of each metabolite versus the isCID energy exhibited a reversed U-shaped curve (Fig. S1e and S1f), especially in the positive mode. This indicates that 
there are optimal and consistent settings across a wide variety of molecules for consistently creating fragments that facilitate identification. We further evaluated these setting with respect to intensity loss of precursor ion and as expected, the intensity of the precursor ion decreases with an increase of isCID energy (Fig. S1g and S1h).

Based on the results observed, we selected $40 \mathrm{eV}$ and $30 \mathrm{eV}$ isCID energy as the eISA condition in negative and positive mode, respectively, with all other parameters fixed. Compared with the original in-source condition (isCID energy is $0 \mathrm{eV}$ ), the $\mathrm{MS}^{1}$ spectra acquired at $40 \mathrm{eV}$ isCID energy in the negative mode shows better fragmentation pattern: $37 \%$ increase in median percentage of fragments (up to 100\% in over half cases), 15\% increase in median match factor, and 3.2 times increase in the median intensity of $1^{\text {st }}$ top fragment ion (Fig. S1a,c,e). Further, as compared with higher isCID energies, a more constant precursor ion intensity (a median loss of only $9 \%$ ) is observed when isCID energy is $40 \mathrm{eV}$ (Fig. S1g). In the positive mode, $\mathrm{MS}^{1}$ spectra at $30 \mathrm{eV}$ isCID energy has a close median match factor and major fragment ion intensity with the original in-source fragmentation condition, but it has higher median percentage of fragments, $96 \%$, which is close to what was observed at higher isCID energies (100\% at $40 \mathrm{eV}$ and higher energies) (Fig. S1b,d,f). More importantly, the median loss of precursor ion intensity at this condition is only $7 \%$ lower, however, this number is $47 \%$ at $40 \mathrm{eV}$ isCID energy, although significantly improved fragmentation data was achieved (Fig. S1h). These results indicate that it is possible to generate in-source fragments comparable with higher energy MS/MS spectra by enhancing ISF condition in the ESI source, while maintaining the intensity of precursor ion.

\section{REFERENCES}

(1) Warth, B.; Raffeiner, P.; Granados, A.; Huan, T.; Fang, M.; Forsberg, E. M.; Benton, H. P.; Goetz, L.; Johnson, C. H.; Siuzdak, G. Metabolomics reveals that dietary xenoestrogens alter cellular metabolism induced by palbociclib/letrozole combination cancer therapy. Cell Chem Biol 2018, 25, 291-300 e293.

(2) Domingo-Almenara, X.; Montenegro-Burke, J. R.; Guijas, C.; Majumder, E. L.; Benton, H. P.; Siuzdak, G. Autonomous METLIN-guided in-source fragment annotation for untargeted metabolomics. Anal Chem 2019, 91, 3246-3253.

(3) Kim, S.; Zhang, X. Comparative analysis of mass spectral similarity measures on peak alignment for comprehensive two-dimensional gas chromatography mass spectrometry. Comput Math Methods Med 2013, 2013, 509761.

(4) Purvine, S.; Eppel, J. T.; Yi, E. C.; Goodlett, D. R. Shortgun collision-induced dissociation of peptides using a time of flight mass analyzer. Proteomics 2003, 3, 847-850.

(5) Ramos, A. A.; Yang, H.; Rosen, L. E.; Yao, X. Tandem parallel fragmentation of peptides for mass spectrometry. Anal Chem 2006, 78, 6391-6397 
Table S1. Absolute (relative) intensities comparison (including both precursor ions and fragments) for metabolites across different insource fragmentation conditions and METLIN library at both positive mode (1-palmitoyl-sn-glycero-3-phosphocholine and cytidine monophosphate) and negative mode (glucose-6-phosphate and uridine)

\begin{tabular}{|c|c|c|c|c|c|c|c|}
\hline \multirow{3}{*}{ analyte } & \multirow{3}{*}{$\mathrm{m} / \mathrm{z}$} & \multicolumn{5}{|c|}{ absolute (relative) intensity } & \multirow{3}{*}{$\begin{array}{l}\text { relative intensity } \\
\text { METLIN (20 eV) }\end{array}$} \\
\hline & & \multicolumn{2}{|c|}{ dry gas temperature $\left({ }^{\circ} \mathrm{C}\right)$} & \multicolumn{3}{|c|}{ is CID energy (eV) } & \\
\hline & & 180 & 240 & 0 & 20 & 40 & \\
\hline precursor ion & 496.34 & 9896886 & 9789667 & 9896886 & 9289506 & 8081574 & \\
\hline fragment 1 & 104.105 & 0 & 0 & 0 & 0 & $20488(100)$ & 54 \\
\hline fragment 2 & 184.069 & 0 & 0 & 0 & 0 & $11562(56)$ & 100 \\
\hline fragment 3 & 478.319 & 14480 & 15260 & 14480 & 11520 & $13658(67)$ & 10 \\
\hline \multicolumn{8}{|c|}{ cytidine monophosphate $(\mathrm{ESI}(+))$} \\
\hline precursor ion & 324.053 & 133396 & 145672 & 133396 & 139096 & 86498 & \\
\hline fragment 1 & 97.029 & $5142(0.3)$ & $5230(0.3)$ & $5142(0.3)$ & $7032(0.3)$ & $15258(0.4)$ & 5 \\
\hline fragment 2 & 112.048 & $1814604(100)$ & $1867800(100)$ & $1814604(100)$ & $2120736(100)$ & 4135146 (100) & 100 \\
\hline \multicolumn{8}{|c|}{ glucose-6-phosphate (ESI(-)) } \\
\hline precursor ion & 259.022 & 454652 & 468930 & 454652 & 475116 & 329692 & \\
\hline fragment 2 & 96.9693 & $57424(60)$ & $56390(59)$ & $57424(60)$ & $49550(52)$ & $47024(100)$ & 100 \\
\hline fragment 3 & 138.978 & $95464(100)$ & $96480(100)$ & $95464(100)$ & $95368(100)$ & $24776(53)$ & 8 \\
\hline \multicolumn{8}{|l|}{ uridine (ESI(-)) } \\
\hline precursor ion & 243.062 & 3674204 & 3756890 & 3674204 & 3928470 & 3615684 & \\
\hline fragment 1 & 66.0347 & 0 & & 0 & 0 & $7134(3)$ & 15 \\
\hline fragment 2 & 82.0301 & 0 & & 0 & 0 & $18696(8)$ & 37 \\
\hline fragment 3 & 110.025 & 41574 (100) & $42750(100)$ & $41574(100)$ & 40960 (100) & $242978(100)$ & 100 \\
\hline fragment 4 & 122.024 & 0 & & 0 & 0 & $14958(6)$ & 11 \\
\hline fragment 5 & 152.034 & $16796(40)$ & $17840(42)$ & $16796(40)$ & $21004(51)$ & $75444(31)$ & 8 \\
\hline fragment 6 & 153.03 & $13222(32)$ & $14630(34)$ & $13222(32)$ & $18756(46)$ & $62994(26)$ & 6 \\
\hline
\end{tabular}


Table S2. Absolute (relative) intensities comparison of both precursor ions and fragments of tyrosine at different levels acquired with QTOF DDA (20 eV) and eISA techniques

\begin{tabular}{|c|c|c|c|c|c|c|c|c|c|c|c|c|}
\hline \multirow{3}{*}{ tyrosine ESI(-) } & & \multicolumn{10}{|c|}{ absolute (relative) intensity } & \multirow{3}{*}{$\begin{array}{l}\text { relative intensity } \\
\text { METLIN (20 eV) }\end{array}$} \\
\hline & & \multicolumn{5}{|c|}{ QTOF DDA } & \multicolumn{5}{|c|}{ elSA } & \\
\hline & & $1 \mathrm{~nm}$ & $10 \mathrm{~nm}$ & $100 \mathrm{~nm}$ & $1 \mu \mathrm{m}$ & $10 \mu \mathrm{m}$ & $1 \mathrm{~nm}$ & $10 \mathrm{~nm}$ & $100 \mathrm{~nm}$ & $1 \mu \mathrm{m}$ & $10 \mu \mathrm{m}$ & \\
\hline Precursor ion & 180.06 & 292 & 272 & 458 & 302 & 1250 & 1228 & 984 & 1542 & 3714 & 13146 & - \\
\hline fragment 1 & 72.007 & $162(100)$ & 0 & $160(80)$ & 0 & 0 & 0 & 0 & 0 & 0 & 0 & 42 \\
\hline fragment 2 & 93.033 & 0 & 0 & 0 & $236(54)$ & 0 & 0 & $172(73)$ & 0 & 0 & $254(21)$ & 27 \\
\hline fragment 3 & 119.05 & 0 & $166(100)$ & $200(100)$ & $438(100)$ & $2870(100)$ & $438(100)$ & $218(92)$ & $190(93)$ & $410(55)$ & $1234(100)$ & 100 \\
\hline fragment 4 & 163.04 & $146(90)$ & 0 & $170(85)$ & $246(56)$ & $694(24)$ & $394(90)$ & $236(100)$ & $204(100)$ & $748(100)$ & $660(54)$ & 25 \\
\hline
\end{tabular}


Table S3. Instrumental sensitivity comparison of the quadrupole time of flight mass spectrometry (QTOF) at three different modes with select metabolites.

\begin{tabular}{lcccccc}
\hline \multirow{2}{*}{ analyte } & \multirow{2}{*}{ mode } & \multirow{2}{*}{ target ion } & \multirow{2}{*}{$\mathrm{RT}^{\mathrm{a}}(\mathrm{min})$} & \multicolumn{3}{c}{ LOD $^{\mathrm{b}}(\mathrm{nM})$} \\
\cline { 5 - 7 } & & & & $\mathrm{eISA}$ & QTOF DIA & QTOF DDA \\
\hline tyrosine & $\mathrm{ESI}(-)$ & 180.0666 & 17.3 & 1 & 1 & 100 \\
inosine & $\mathrm{ESI}(-)$ & 267.0735 & 16.6 & 1 & 1 & 1 \\
UMP & $\mathrm{ESI}(-)$ & 323.0286 & 31.8 & 1 & 1 & 1 \\
oxidized glutathione & $\mathrm{ESI}(-)$ & 611.1447 & 32.4 & 1 & 1 & 1 \\
glutamine & $\mathrm{ESI}(+)$ & 147.0764 & 6.9 & 1000 & 1000 & 10000 \\
histidine & $\mathrm{ESI}(+)$ & 156.0766 & 6.7 & 1 & 1 & 100 \\
phenylalanine & $\mathrm{ESI}(+)$ & 166.0863 & 7.7 & 1 & 1 & 100 \\
oxidized glutathione & $\mathrm{ESI}(+)$ & 613.1592 & 7.1 & 10 & 100 & 100 \\
\hline
\end{tabular}

${ }^{\mathrm{a}} \mathrm{RT}$ : retention time; ${ }^{\mathrm{b} L O D}$ : limit of detection. 
Table S4. Fragmentation pattern comparison between mass spectra acquired in the eISA mode and QTOF DIA mode $(20 \mathrm{eV})$

\begin{tabular}{|c|c|c|c|c|c|c|}
\hline \multirow{2}{*}{ no. } & \multicolumn{3}{|c|}{ ESI (-) } & \multicolumn{3}{|c|}{ ESI (+) } \\
\hline & name & score $^{a}$ & M.F.b & name & score & M.F \\
\hline 1 & aspartate & $4 / 4$ & 96 & & & \\
\hline 2 & malate & $4 / 4$ & 40 & & & \\
\hline 3 & adenine & $2 / 4$ & 100 & adenine & $2 / 3$ & 100 \\
\hline 4 & hypoxanthine & $2 / 3$ & 100 & hypoxanthine & $2 / 5$ & 98 \\
\hline 5 & sulfoacetic acid & $2 / 2$ & & & & \\
\hline 6 & glutamine & $8 / 9$ & 93 & glutamine & $3 / 3$ & 61 \\
\hline 7 & glutamate & $2 / 2$ & & glutamate & $3 / 3$ & 84 \\
\hline 8 & methionine & $1 / 1$ & & methionine & $6 / 7$ & 55 \\
\hline 9 & xanthine & $2 / 2^{*}$ & 100 & xanthine & $1 / 4$ & 100 \\
\hline 10 & aminoadipic acid & $5 / 5 *$ & 16 & aminoadipic acid & $4 / 5$ & 81 \\
\hline 11 & phenylalanine & $4 / 5$ & 89 & phenylalanine & $2 / 2$ & \\
\hline 12 & phosphoenol pyruvate & $1 / 1$ & & & & \\
\hline 13 & uric acid & $4 / 4$ & 99 & & & \\
\hline 14 & glyceraldehyde-3P & $2 / 2$ & & & & \\
\hline 15 & arginine & $1 / 1$ & & arginine & $4 / 4$ & 54 \\
\hline 16 & tyrosine & $3 / 4$ & 92 & tyrosine & $8 / 9$ & 80 \\
\hline 17 & acetyl-glutamic acid & $5 / 6$ & 33 & acetyl-glutamic acid & $4 / 4$ & 91 \\
\hline 18 & citric acid & $5 / 5$ & 97 & & & \\
\hline 19 & tryptophan & $4 / 4$ & 96 & tryptophan & $7 / 7$ & 62 \\
\hline 20 & myristic acid & $0 / 0$ & & & & \\
\hline 21 & ribose-5-phosphate & $3 / 3$ & 55 & & & \\
\hline 22 & cytidine & $5 / 5$ & 100 & cytidine & $1 / 1$ & \\
\hline 23 & uridine & $6 / 6$ & 100 & uridine & $1 / 3$ & 100 \\
\hline 24 & palmitic acid & $0 / 0$ & & palmitic acid & $12 / 12$ & 78 \\
\hline 25 & glucose-6-phosphate & $3 / 3$ & 94 & glucose-6-phosphate & $5 / 6$ & 97 \\
\hline 26 & fructose-6-phosphate & $3 / 3$ & 94 & fructose-6-phosphate & $2 / 2$ & \\
\hline 27 & adenosine & $1 / 1$ & & adenosine & $1 / 1$ & \\
\hline 28 & inosine & $1 / 1$ & & inosine & $1 / 1$ & \\
\hline 29 & saccharopine & $7 / 13^{*}$ & 98 & saccharopine & $2 / 4$ & 68 \\
\hline 30 & linoleic acid & $0 / 0$ & & & & \\
\hline 31 & oleic acid & $0 / 0$ & & oleic acid & $23 / 25$ & 83 \\
\hline 32 & guanosine & $3 / 3$ & 6 & guanosine & $1 / 1$ & \\
\hline 33 & EPA & $2 / 7$ & 25 & & & \\
\hline 34 & CMP & $3 / 3$ & 74 & CMP & $2 / 2$ & \\
\hline 35 & UMP & $6 / 6$ & 80 & UMP & $2 / 2$ & \\
\hline 36 & fructose-1,6-bisphosphate & $5 / 5$ & 94 & & & \\
\hline 37 & AMP & $3 / 3$ & 74 & AMP & $1 / 1$ & \\
\hline 38 & IMP & $3 / 5$ & 84 & IMP & $1 / 2$ & \\
\hline 39 & GMP & $3 / 3$ & 58 & GMP & $1 / 2$ & \\
\hline 40 & UDP & $4 / 9$ & 16 & & & \\
\hline 41 & ADP & $5 / 7$ & 30 & $A D P$ & $2 / 3$ & 100 \\
\hline 42 & GDP & $4 / 6$ & 23 & GDP & $1 / 1$ & \\
\hline 43 & $P G(16: 0 / 0: 0)$ & $1 / 3$ & 98 & PG(16:0/0:0) & $2 / 3$ & 100 \\
\hline 44 & lysoPC(16:0) & $1 / 3$ & 100 & IysoPC(16:0) & $1 / 3$ & 25 \\
\hline 45 & GTP & $9 / 9 *$ & 46 & & & \\
\hline 46 & UDP-galactose & $3 / 6$ & 14 & & & \\
\hline 47 & UDP-N-acetyl-glucosamine & $5 / 8$ & 80 & & & \\
\hline 48 & oxidized glutathione & $10 / 10$ & 76 & oxidized glutathione & $5 / 9$ & 76 \\
\hline 49 & dioleoylphosphatidic acid & $2 / 3^{*}$ & 73 & & & \\
\hline 50 & acetyl-CoA & $4 / 4$ & 78 & acetyl-CoA & $4 / 6$ & 71 \\
\hline
\end{tabular}

${ }^{a}$ score: ratio score; ${ }^{b}$ M.F.: match factor; ${ }^{*}$ : the number of fragments found in the QTOF DIA mode is less than that recorded in the $20 \mathrm{eV}$ mass spectra in the METLIN database; the calculation of both score and M.F. was based on the $20 \mathrm{eV}$ MS/MS spectra produced in the QTOF DIA mode. 

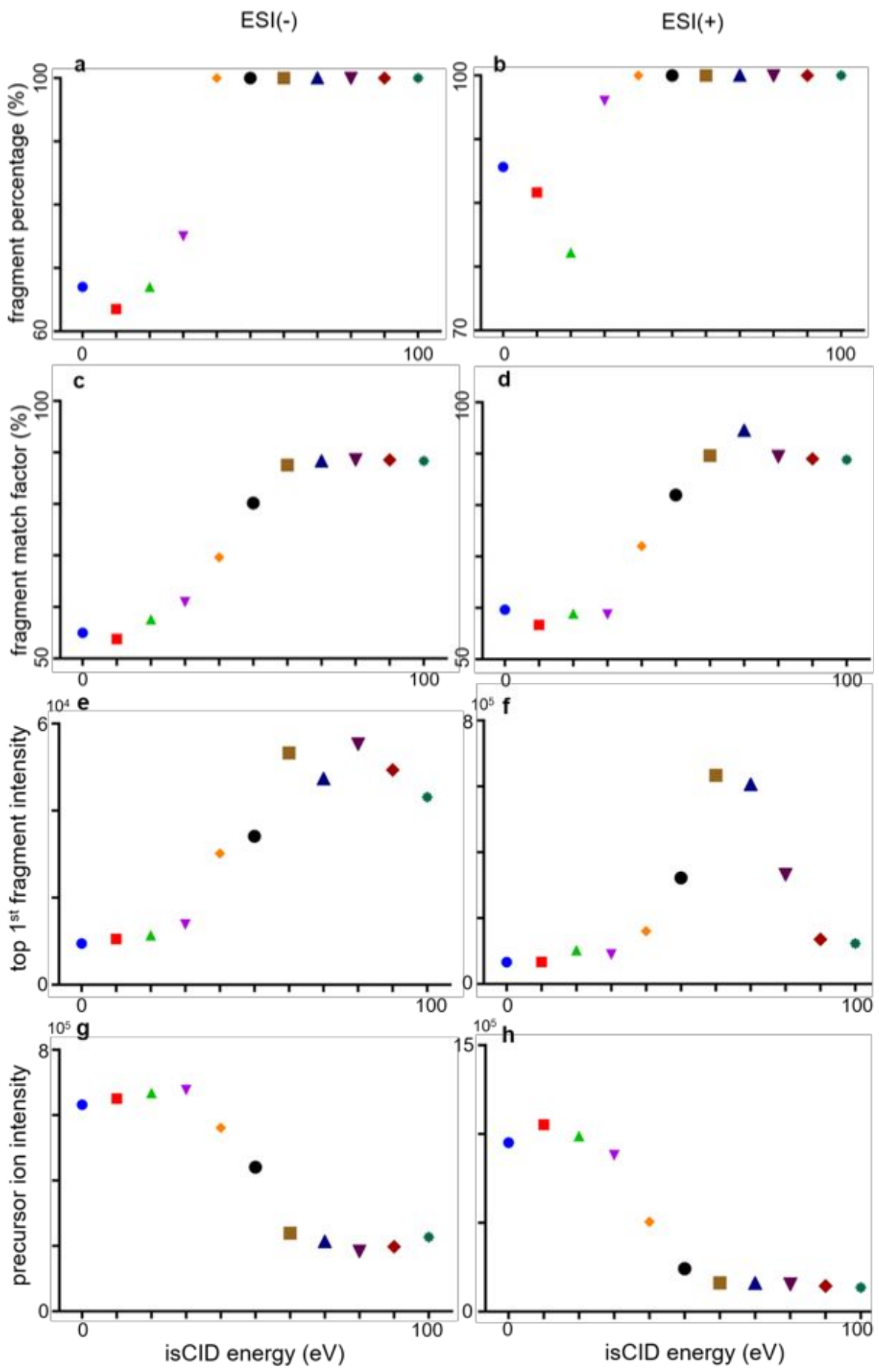

Figure S1. Panels (a) and (b) plot the relationship between median fragment percentage and isCID energy. Panels (c) and (d) plot the relationship between median fragment match factor versus isCID energy. Panels (e) and (f) plot the relationship between median intensity of the major ( $1^{\text {st }}$ top) fragment ion versus isCID energy. Panels $(\mathrm{g})$ and $(\mathrm{h})$ plot the relationship between median intensity of the precursor ion versus isCID energy. Panels (a), (c), (e), and (g) were observed at negative mode (ESI(-)) and others were observed at positive mode (ESI $(+))$. Both fragment percentage and match factor were calculated versus the $+/-20 \mathrm{eV} \mathrm{MS/MS} \mathrm{spectra} \mathrm{in}$ the METLIN database. 

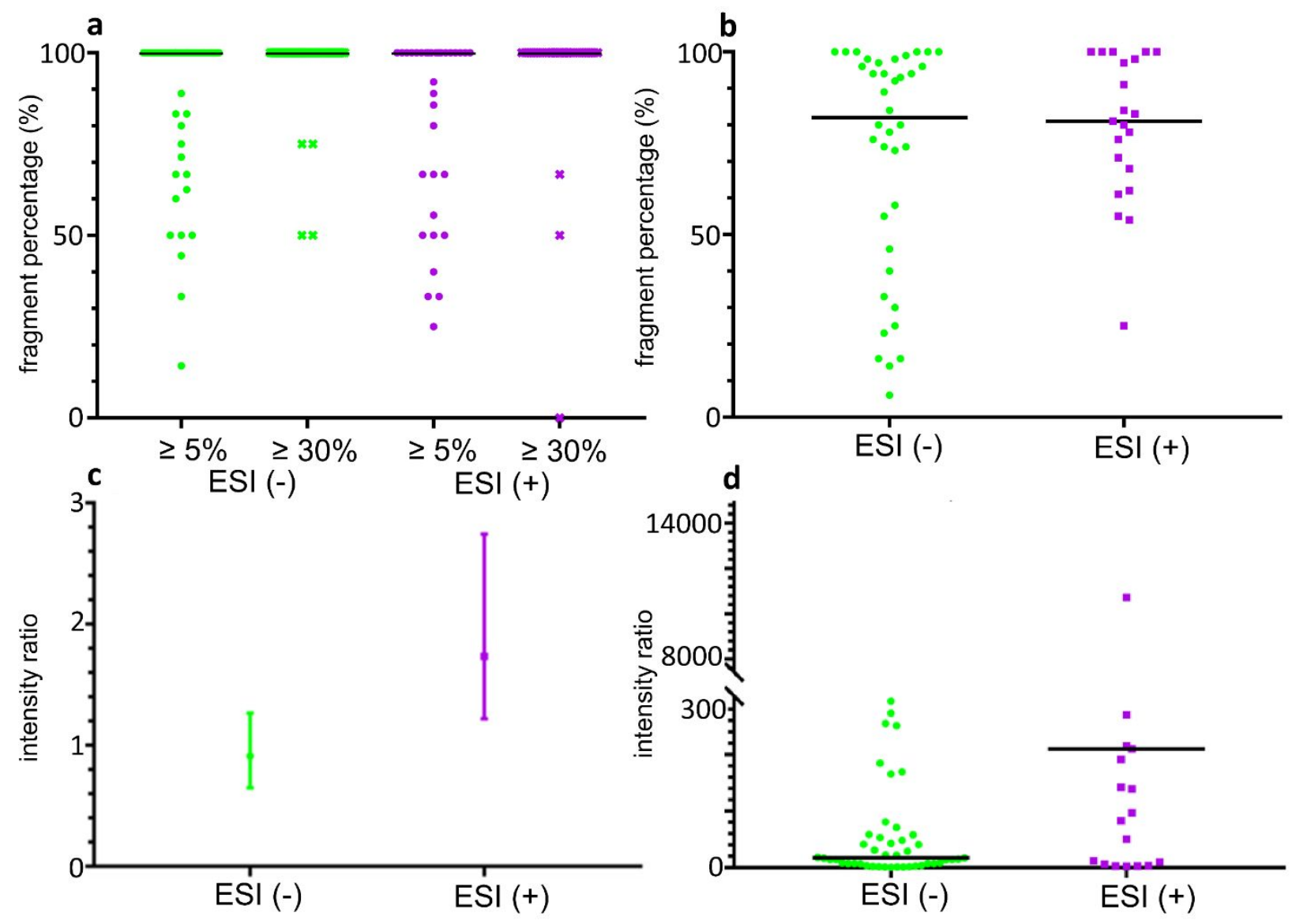

Figure S2. The percentage of fragment produced at eISA mode versus QTOF DDA mode increases with the increase of relative intensity of fragments (from $\geq 5 \%$ to $\geq 30 \%$ ) considered at both ionization modes with black line representing media (a). Panel (b) shows the vertical scatter plot with median fragment match factor (eISA vs. QTOF DDA) across different polarity modes. Panel (c) shows the absolute intensity ratio (median plus 95\% CI) of fragments generated by eISA versus those generated with QTOF DDA technique. Panel (d) shows the absolute intensity ratio (median plus $95 \% \mathrm{CI}$ ) of precursor ions generated by eISA versus those generated with QTOF DDA technique. MS/MS spectra in the QTOF DDA mode was generated at $20 \mathrm{eV}$. 

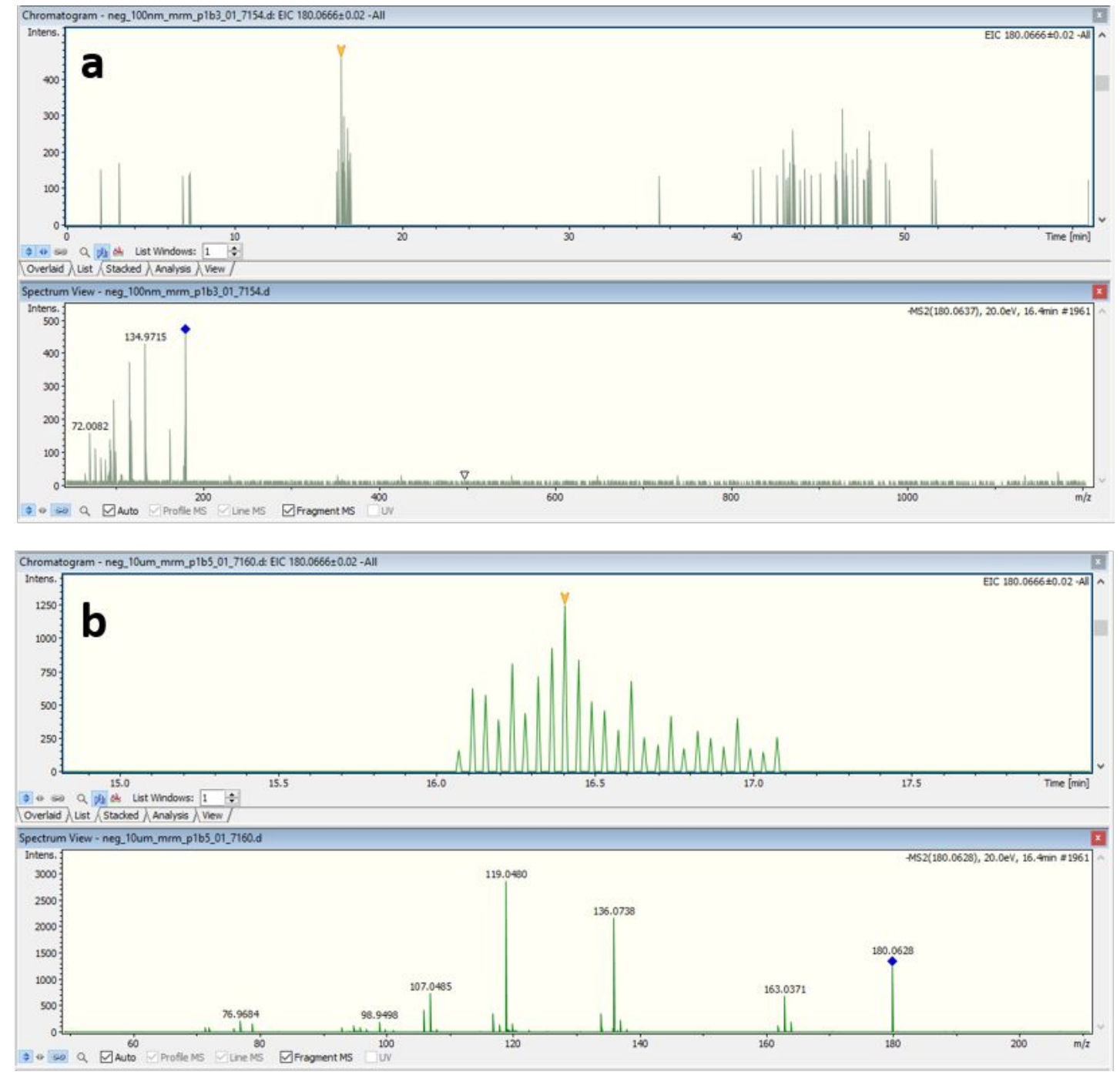

Figure S3. MS/MS spectra of tyrosine acquired at $100 \mathrm{nM}$ (a) and $10 \mu \mathrm{M}$ (b) using QTOF DDA mode (ESI(-)). 